\title{
Classification and learning of distributed stimulus sets
}

\author{
BERT ZIPPEL and JOSEPH KARPIENIA \\ City University of New York, Hunter College, New York, New York 10021
}

\begin{abstract}
One compact and four different types of distributed stimulus representations varying in three attributes and two values per attribute were generated. Eighty-four students partitioned a set of stimuli in any way they wished. Representations using different attributes to convey stimulus information were grouped similarly as distinct from a Morse code type of representation that varied values of the same attribute. In a second experiment, 27 students were required to learn one of three classification rules employing one of three different types of distributed stimulus sets. Performance was best when the imposed rule corresponded to previous sorting preferences. However, the three types of distributed stimulus sets were classified and learned more like compact stimuli than like the Morse code type of stimulus set.
\end{abstract}

The sorting of sets of pictorial geometric stimuli is influenced by principles of set construction (Zippel, 1968), and these sorting behaviors predict the ease of learning of imposed partitions on stimulus groups (Zippel, 1969). The different principles used to generate stimulus groups in previous studies were only two possibilities of a larger set. In the present studies, the classification and learning of stimuli generated by other possible principles were investigated.

In a previous classification study (Zippel, 1968), stimuli were generated as either compact or distributed sets (Shepard, Hovland, \& Jenkins, 1961). Compact stimulus sets were generated by randomly fixing the value of three of the attributes. For example, a set could contain a large blue thick-bordered form as a base with other forms being large or small, blue or red, and thick or thin bordered. Distributed stimulus sets were generated by randomly fixing values of the same 11 attributes for one stimulus element and then varying values of one of the attributes across three elements that constituted one stimulus form. For example, a distributed set could contain the same large blue thickbordered element described above with color changing from element to element. This would produce a series of three large thick-bordered elements that differed only in whether each element in the form was blue or red. The compact sets were comparable to those of Shepard et al. (1961). The distributed sets were comparable to those used by Fallon and Battig (1964) and Metzger (1958), but they differed from the Shepard et al. (1961) distributed stimuli. Although all of the distributed stimuli used by Shepard et al. (1961) involved a stimulus form of three successive elements, those authors chose to vary either a different attribute for each element or the same attribute across all three elements, but with

Requests for reprints should be sent to Bert Zippel, Box 389, Hunter College of The City University of New York, 695 Park Avenue, New York, New York 10021. the geometric shape of each element position also being different. The shape of the element was redundant with the position in which the element appeared. From the two types of distributed representation used by Shepard et al. (1961) and the third type of representation used by Fallon and Battig (1964), Metzger (1958), and Zippel (1968, 1969), a two-factor basis of constructing distributed stimulus representations was developed. In each position the same or a different attribute could be varied. Also, all of the other attributes in an element could have the same values across positions, or one of the attributes of an element could take on a value that was redundant with the position of the element in the sequence. The four representations are depicted in Figure 1. They have been named the Metzger, Shepard B, Shepard C, and double-redundant types to reflect previous usage.

\section{EXPERIMENT 1}

\section{Method}

Subjects. The participants of this study were 84 undergraduate students enrolled in various psychology courses who participated in groups of from 15 to 35 members.

Procedure. Three different compact stimulus sets and three Metzger sets were generated according to the procedure mentioned above. Two different sets each of the other three types of distributed forms were generated according to similar procedures. Copies of each form of the resulting stimulus sets were drawn on $3 \times 5$ in. index cards, and the eight cards for each set were placed in envelopes along with a set of instructions and a response sheet for the student to indicate the classification. A two-digit number was randomly assigned to each stimulus form and placed on the back of the card. Envelopes were randomly handed out to all members of each class that participated. Students were instructed to sort the cards in any way they wished and to use the numbers on the back of the cards to indicate the groupings.

\section{Results and Discussion}

In the previous classification study (Zippel, 1968), 
it was found that most people sorted the sets into two groups of four members each (SHJ partitions), four groups of two members each ( $\mathrm{Z}$ partitions), or according to a frequency distribution partition (FD) that classed the forms according to the number of times that one of the values of the varied attribute appeared, from zero to three. These partitions were found to be adequate to describe the results of this study. The proportion of unclassified sortings for the different representations showed differences that were consistent with the previous study in that the distributed sets produced unclassified sortings, while the compact sets produced none. A few of the unclassified sortings were due to students' not listing all eight members of the sets, but most were due to students' listing all eight set members without indicating partitions among subsets.

Table 1 presents the total number of examples of various partitions used by the students to sort the stimulus sets. The combined SHJ and Z sortings compared with all other sortings for each representation

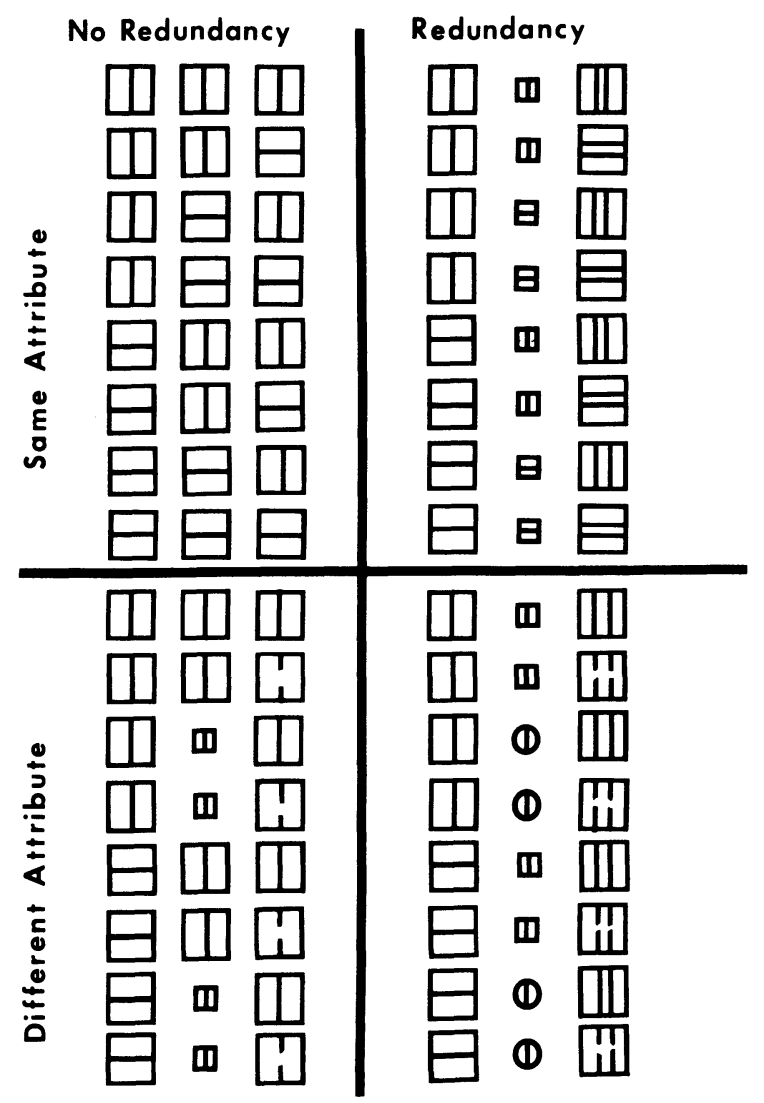

Figure 1. Sample sets of stimuli for four types of distributed stimulus sets. For the top sets the same attribute is varied to convey the information. For the bottom sets a different attribute conveys the information. For the left sets the attributes other than the one conveying information have the same values across positions. For the right sets one of the attributes varies in each position to be redundant with position. The top left set is a Metzger set; the top right, Shepard B; the bottom left, Shepard C; and the bottom right, a double-redundant set.
Table 1

Sorting for Five Different Stimulus Representations

\begin{tabular}{|c|c|c|c|c|c|}
\hline \multirow{3}{*}{$\begin{array}{c}\text { Partition } \\
\text { Type }\end{array}$} & \multicolumn{5}{|c|}{ Stimulus Representation } \\
\hline & \multirow{2}{*}{$\begin{array}{l}\text { Com- } \\
\text { pact }\end{array}$} & \multirow{2}{*}{$\begin{array}{c}\text { Double } \\
\text { Redundant }\end{array}$} & \multicolumn{2}{|c|}{ Shepard } & \multirow[b]{2}{*}{ Metzger } \\
\hline & & & $\mathrm{C}$ & B & \\
\hline SHJ or Z & 15 & 10 & 13 & 9 & 11 \\
\hline FD & 0 & 0 & 0 & 2 & 9 \\
\hline Unclassed & 0 & 4 & 1 & 3 & 7 \\
\hline
\end{tabular}

show significantly different distributions among the five stimulus types $\left[\chi^{2}(4)=20.73, p<.01\right]$. No significant differences, using Fisher's exact test of probability, were found among the double-redundant, Shepard $\mathrm{C}$, and compact representations or between the Metzger and Shepard B sets. The combined data for the compact, double-redundant, and Shepard C types of sets compared with the Metzger and Shepard B sets were significantly different $\left[\chi^{2}(1)=13.6, p<.01\right]$.

Most students used either an SHJ or Z partition to classify the compact, double-redundant, or Shepard C representations. The Metzger and Shepard B representations elicited FD as well as SHJ or Z sortings. The Metzger form of representation emphasizes repeating value changes of the same attribute. The Shepard B representation also seems to offer strong cues that emphasize the different positions. Perhaps this Shepard B form of representation has not been clearly developed in this study. There is one cue that differentiates Position 1 from the other two positions, but there are at least two cues that differentiate Position 2 from Position 3. In general, it appears that the presence of such cues interferes with the perception of the relevant varying attribute, thus diminishing the probability of an FD partition.

In general, the data of the present study suggest that the difference between compact and distributed forms of geometric stimuli are of much less importance in predicting classification than whether the variation in same or different attributes conveys the essential information about differences in stimulus forms.

\section{EXPERIMENT 2}

This experiment was conducted to determine whether individuals would make fewer errors in learning to classify the Shepard B, Shepard C, or double-redundant sets according to a simple two-dimensional (Z1) rule than according to an $F D$ rule. The $\mathrm{Z} 1$ rule requires disregarding one attribute and sorting on the basis of the conjunction of the values of the other two attributes. This rule is spontaneously used more often than other possible partitions in classifying compact stimuli. The FD rule requires classifying by the number of differences in each stimulus as compared with an anchor. This rule is spontaneously used more often than any other in classifying Metzger stimulus sets (Zippel, 1968). In addition, according to the previous 
results of Zippel (1969), a paired associates (PA) task should produce an error rate between that of the $\mathrm{Zl}$ and FD rules.

\section{Method}

Subjects. Three volunteer undergraduate psychology students who had not previously participated in any similar research were randomly assigned to one of nine different conditions, for a total of 27 participants.

Procedure. Three different stimulus sets each of the Shepard B, Shepard C, and double-redundant type were generated according to the principles of the first study. Each stimulus set was replicated three times, and then each stimulus was stapled on a $3 \times 5$ in. index card. Each identical set of eight cards was placed in random order in a different card holder. A student was then presented the three card holders, one at a time, in random order, for 160 presentations or 16 consecutive responses without error, whichever came first. Each participant was requested to learn the $\mathrm{FD}, \mathrm{PA}$, or $\mathrm{Z1}$ rule.

For the $\mathrm{Z} 1$ rule, students were asked to group the cards into four groups of two members each. A card with four printed letters was given to the student to serve as responses. Each letter represented a pair of cards. The correct letter response for the card was announced after the student responded.

For the FD rule, the student was given four letters that would serve as responses and was instructed to group the eight cards into two groups of one member and two groups of three members each. The student was not told which response letters were associated with the single-member or triple-member groups. The rest of the procedure was the same as in $\mathrm{Z} 1$ conditions. The correspondence between letters and cards was determined by randomly associating one letter with any one card and then using a second letter to correspond with the three cards that differed in one way from the anchor. A third letter was associated with the three cards that differed in two ways from the anchor, and the fourth letter was associated with the card that differed in three ways from the anchor.

For the PA rule, the student was given eight letters that would serve as responses and was instructed to match each letter with a stimulus card. The rest of the procedure was the same as in the other learning conditions.

\section{Results}

Most students did not reach the criterion of 16 consecutive errorless presentations within 160 stimulus presentations. Thus, the errors committed were used for analysis. In the four response alternative conditions (Z1 and FD), the probability of a correct response by chance alone was .25 , compared with .125 for the eight response PA conditions. Thus, an error measure was taken that represented the proportionate difference between total errors made and chance errors expected for each participant.

Across all stimulus types, the $\mathrm{Z} 1$ rule produced the fewest errors, followed by the PA rule, and then the FD rule $[F(2,18)=9.69, p<.01]$. Table 2 provides error rates for all nine experimental conditions. A Scheffé test of all possible pairs of rules learned regardless of type of stimulus display showed no significant difference between $\mathrm{Z} 1$ rule learning and PA learning. The differences between FD rule learning and both $\mathrm{Z} 1$ rule learning $[F(2,24)=17.37, p<.01]$ and PA rule learning $[F(2,24)=10.98, p<.05]$ were significant. The dif-
Table 2

Proportionate Errors for Learning Partition Rules as a Function of Stimulus Displays

\begin{tabular}{lccc}
\hline & \multicolumn{3}{c}{ Partition Rules } \\
\cline { 2 - 4 } Stimulus & ZI & PA & FD \\
\hline Displays & .38 & .62 & .78 \\
Double Redundant & .63 & .59 & .80 \\
Shepard C & .59 & .74 & .83 \\
Shepard B & &
\end{tabular}

ferences due to type of stimulus display were not significant. No interactions approached significance.

\section{Discussion}

When the $\mathrm{Z} 1$ and FD rules were imposed on each of the three types of distributed stimulus sets, the $\mathrm{Z} 1$ rule produced better learning. Thus, except for the Shepard B sets, the present studies confirm and generalize the finding that ease of learning imposed classifications can be predicted by spontaneous classification of these stimulus materials. The $\mathrm{Z1}$ rule was shown to be appropriate for classification of distributed stimuli except for the Metzger type. For both the Metzger and the Shepard B type of distributed stimulus sets, some students sorted according to SHJ or Z rules. No students used FD rules to sort any of the other three types of distributed sets. Even though the Shepard B sets were classed with the Metzger sets in Experiment 1 on the basis that some students used FD sortings, the predominant sorting rule was $\mathrm{SHJ}$ or $\mathrm{Z}$ rule. Thus, the easier learning of the $\mathrm{Z} 1$ than the FD rule for the Shepard B condition in this experiment is not surprising.

There is one important limitation to these findings. In the present learning study, the correspondence between labels and individual stimulus set members was determined by randomly choosing one stimulus as an anchor. For all distributed stimulus sets, some stimuli would better serve as an anchor than others. For example, in generating Shepard C and Metzger sets, one stimulus pattern is identical in all three positions; such stimuli might better serve as a perceptual anchor than one that is randomly chosen. It would be advisable to study the application of the FD rule to the different distributed sets as a function of the nature of the label-stimulus correspondences. It appears that the FD rule is applied only to that form of distributed stimulus, Metzger, that relies on only one cue to convey differences in positions. If the informational cue is different in each position as in the Shepard C and double-redundant sets, or each element in the stimulus array contains a cue redundant with positions as in Shepard B, then the $\mathrm{Z1}$ rule is easier to learn.

\section{REFERENCES}

Fallon, D., \& Battig, W. F. Role of difficulty in rote and concept learning. Journal of Experimental Psychology, 1964, 68, 85-88.

Metzger, R. A. A comparison between rote learning and concept formation. Journal of Experimental Psychology, 1958, 56, 226-231.

Shepard, R. N., Hovland, C. I., \& Jenkins, H. M. Learning and memorization of classifications. Psychological Monographs, 1961, 75(13, Whole No. 517).

ZIPPEL, B. Free classification of compact and distributed stimulus sets. Proceedings, 76th Annual Convention of the American Psychological Association, 1968, 3, 45-46.

$\mathrm{Z}_{\text {IPPEL}}$, B. Unrestricted classification behavior and learning of imposed classifications in closed, exhaustive stimulus sets. Journal of Experimental Psychology, 1969, 82, 493-498.

(Received for publication December 5, 1979.) 